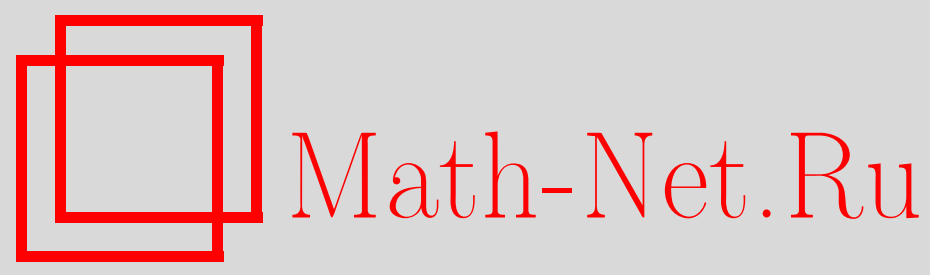

У. А. Розиков, Представимость деревьев и их некоторые приложения, Матем. заметки, 2002, том 72, выпуск 4, 516527

DOI: https://doi.org/10.4213/mzm441

Использование Общероссийского математического портала Math-Net.Ru подразумевает, что вы прочитали и согласны с пользовательским соглашением http://www . mathnet.ru/rus/agreement

Параметры загрузки:

IP : 52.87 .193 .239

26 апреля 2023 г., 13:04:00

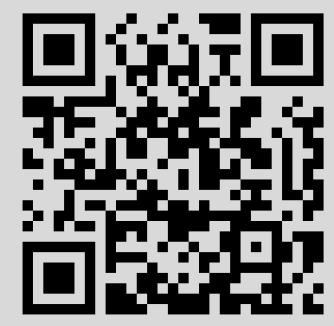


УДК $519.17+530.1$

\title{
ПРЕДСТАВИМОСТЬ ДЕРЕВЬЕВ \\ И ИХ НЕКОТОРЫЕ ПРИЛОЖЕНИЯ
}

\section{У. А. Розиков}

\begin{abstract}
Доказано, что только дерево Кэли представляется как группа свободного произведения конечного числа циклических групп второго порядка. Для остальных деревьев даны их представления как некоторое множество конечных последовательностей, построенных по некоторым рекуррентным соотношениям. Используя эти представления, дано полное описание трансляционно-инвариантных и одного класса периодических гиббсовских мер неоднородной модели Изинга на произвольном дереве. Найдено достаточное условие невозвратности случайного блуждания в случайной среде на произвольном дереве.
\end{abstract}

Библиография: 15 названий.

1. Введение. Деревом назьвается связный граф, не содержащий циклов. Класс деревьев занимает в теории графов особое положение. Это достаточно просто устроенные графы, и многие задачи, весьма сложные в общей ситуации, для деревьев решаются легко.

Деревья открьвались независимо несколько раз. Еще в прошлом веке Г. Кирхгоф ввел деревья и применил их к исследованию электрических цепей, а А. Кэли, перечисляя изомеры насьшенных углеводов, еще раз открыл и первьм исследовал их свойства. Тогда же деревья были введены и исследованы К. Жорданом как чисто математический объект.

В настояшее время на графах типа дерева изучаются многие задачи статистической механики, теории вероятностей, математического анализа и других разделов математики (см., например, [1]-[11]).

Одним из частных случаев дерева является дерево Кәли, т.е. бесконечное дерево, из каждой вершины которого выходит ровно $k+1$ ребер (дерево Кэли порядка $k \geqslant 1$ ). На дереве Кэли изучены модели статистической механики и для них описаны множества периодических гиббсовских мер, а также изучены траектории случайного блуждания в случайной среде в работах [5]-[12].

Для изучения этих задач используется групповое представления дерева Кэли [5]; в этом случае эти задачи формулируются достаточно просто на языке групшы, которая представляет дерево Кэли.

Но к сожалению, произвольное бесконечное дерево не всегда удается представить как группу. 
Цель работы:

1) выделить класс деревьев, которые представляются как групшы, и дать их групповое представление;

2) представить деревья, не имеющие группового представления, как некоторое множество конечных последовательностей, построенных по некоторым рекуррентным соотношениям;

3) описать периодические гиббсовские меры модели Изинга на деревьях, введенных в п. 2 (т.е. на представимых деревьях);

4) определить и изучить траектории случайного блуждания в случайной среде на произвольном дереве.

Эти задачи решаются в пा. $2-5$ соответственно.

2. Представимые деревья. Известно, что всякая группа может быть задана системой определящих соотношений относительно некоторого множество образующих. Обратно, если дано произвольное множество символов (образующих) $M$ и произвольная система соотношений, приравнивающих единице некоторые слова, составленные из символов, входящих в $M$, то всегда можно указать группу, для которой эти соотношения составляют систему определяющих соотношений [13].

Всякая групша может быть задана образуюшими и определяющими соотношениями многими различными способами. Поэтому, хотя определяющие соотношения представляют собою удобньй способ “абстрактного” задания группы, в большинстве случаев о группе, заданной соотношениями, удается сказать очень мало. Например, нельзя установить, конечна ли эта группа или бесконечна, коммутативна она или нет и т.д.

Заметим, что если $G$ - свободная групша и $M$ - ее множество образующих - конечно либо счетно, то $G$ будет счетной, а если $M$ несчетно, то и $G$ будет несчетной [13].

Пусть $(G, M, S)$ - тройка, где $G$ - группа с множеством образующих $M$ и определяющих соотношений $S$.

На $(G, M, S)$ определим структуру графа следующим образом: два слова $x, y \in G$ назовем ближсайшими соседями и соединим ребром, если существует $a \in M$ такое, что $x=y a$ либо $y=x a\left(y=x a^{-1}\right.$ либо $\left.x=y a^{-1}\right)$.

Обозначим через $\Gamma_{G}$ граф групшы $G$. Например, граф свободной групшы с множеством образующих $M$ будет деревом Кэли порядка $2|M|-1$, где | | - число элементов множества.

ОПРЕДЕЛЕНИЕ 1. Дерево Г назовем представимы.м, если оно является графом некоторой группы.

ТеОрема 1. Граф группы $(G, M, S)$ образует дерево тогда и только тогда, когда $S$ содержит только соотношения типа $a^{2}=1, a \in M$. Более того, этот граф совпадает с деревом Кәли.

ДокАЗАтЕЛЬСтво. Необходимость. Пусть $\Gamma_{G}$ является деревом, т.е. не содержит циклов. Так как дерево не содержит петель и кратных ребер, то имеются только равенства $a a^{-1}=1$ (которые не удовлетворяют определению соотношения, см. [13]) и соотношения типа $a^{2}=1$.

Достаточность. Пусть $S$ содержит только соотношения типа $a^{2}=1$. Докажем, что $\Gamma_{G}$ является деревом. Пусть $1 \in G$ - единичный элемент; очевидно, что ближайшими 
соседями этого элемента будут точки из $M$ и обратные элементы каждого элемента $a \in M$ такого, что $a \notin S$, т.е. $a^{2}=1$. Если $a \in S$, то ближайшие соседи точки $a$ будут

$$
a^{2}=1, \quad a b, \quad b \in M \backslash\{a\}, \quad a b^{-1}, \quad b \in M \backslash S .
$$

Если $a \notin S$, то ближайшие соседи точки $a$ будут

$$
a a^{-1}, \quad a b, \quad b \in M, \quad a b^{-1}, \quad b \in M \backslash(S \cup\{a\}) .
$$

Таким образом, если $x \in G$ заканчивается буквой $a \in S$, то ближайшие соседи точки $x$ будут

$$
x a=a_{i_{1}} a_{i_{2}} \ldots a_{i_{n}}, \quad x b, \quad b \in M \backslash\{a\}, \quad x b^{-1}, \quad b \in M \backslash S,
$$

а если $a \notin S$, то

$$
x a^{-1}=a_{i_{1}} a_{i_{2}} \ldots a_{i_{n}}, \quad x b, \quad b \in M, \quad x b^{-1}, \quad b \in M \backslash(S \cup\{a\})
$$

будут ближайшими соседями точки $x$. Следовательно, получается дерево Кэли порядка $2|M|-|S|-1$. Теорема доказана.

\section{СлЕДСТВИЕ 1. Деревья Кәли и только они представимы.}

K сожалению, класс представимых деревьев намного уже, чем множество всех деревьев. Поэтому для произвольного дерева, во-первых, необходимо его пометить, т.е. присвоить его вершинам некоторые метки, во-вторых, на помеченном дереве необходимо определить понятие периодического отображения, которое понадобится для описания периодических гиббсовских мер и изучения случайного блуждания в случайной среде. В п. 3 предлагается одно решение последней проблемы.

3. Разбиение непредставимых деревьев. Пусть $\Gamma$ - бесконечное дерево, т.е. граф без циклов. Предположим, что из каждой точки $x \in \Gamma$ выходит не менее 1 и не более $k_{\Gamma}<+\infty$ ребер.

Обозначим через $V$ множество вершин и через $L$ множество ребер дерева $\Gamma$.

Пусть $x^{0} \in V$ фиксировано. Для $x \in V$ обозначим через $|x|$ число ребер пути, соединяющего $x^{0}$ и $x$. Для $x, y \in V$ обозначим через $d(x, y)$ число ребер кратчайшего пути, соединяющего $x$ и $y$.

Для $x, y \in V$ мы будем писать $x \leqslant y$, если $x$ находится на кратчайшем пути, соединяющем $x^{0}$ и $y$; будем писать $x<y$, если $x \leqslant y$ и $x \neq y$. Если $x \leqslant y$ и $|y|=|x|+1$, то будем писать $x \rightarrow y$. Точку $x^{0}$ назовем корнем дерева $\Gamma$.

Обозначим

$$
S(x)=\{y \in V: x \rightarrow y\}, \quad S_{1}(x)=\{y \in V: d(x, y)=1\} .
$$

Пусть даны натуральные числа

$$
\begin{gathered}
\alpha_{1}, \quad \alpha_{1 i_{1}}, \quad i_{1}=1,2, \ldots, \alpha_{1}, \quad \alpha_{1 i_{1} i_{2}}, i_{2}=1,2, \ldots, \alpha_{1 i_{1}}, \ldots, \ldots, \quad \alpha_{1 i_{1} i_{2} \ldots i_{n-1}}, \ldots . \\
\alpha_{1 i_{1} i_{2} \ldots i_{n}}, \quad i_{n}=1,2, \ldots, \quad \ldots
\end{gathered}
$$

Такие множества натуральных чисел будем назьвать множествами вида $\mathscr{A}$. Для произвольного множества $A$ вида $\mathscr{A}$ определим

$$
\begin{aligned}
K_{A}= & \left\{\left(i_{0}, i_{1}, i_{2}, \ldots, i_{n}\right): i_{0}=1 ; i_{1}=1,2, \ldots, \alpha_{1} ; i_{2}=1,2, \ldots, \alpha_{1 i_{1}} ; \ldots ;\right. \\
& \left.i_{n}=1,2, \ldots, \alpha_{1 i_{1} i_{2} \ldots i_{n-1}} ; n \geqslant 0\right\} .
\end{aligned}
$$

Пусть $B$ - совокупность всевозможных множеств вида $\mathscr{A}$ и $\Lambda$ - множество всевозможных деревьев $\Gamma$. 
ПРЕДЛОЖЕНИЕ 1. Существует взаимно однозначное соответствие $F$ между әлементами $\Lambda$ и $B$.

ДокАзАтЕльСтво. Отображение $F: \Lambda \rightarrow B$ строим следующим образом. Для любого $Г \in \Lambda$ положим

$$
F(\Gamma)=\{|S(x)|, x \in \Gamma\} .
$$

Очевидно, что правая часть есть множество вида $\mathscr{A}$ и это соответствие взаимно однозначное. Предложение доказано.

Обозначим через $A(\Gamma)$ множество вида $\mathscr{A}$, соответствующее дереву $\Gamma$.

ПРЕДЛОЖЕНИЕ 2. Для любого дерева Г существует взаимно однозначное соответствие мехду әлементами мнохества $K_{A(\Gamma)}$ и множсеством вериин $V$ дерева Г.

ДокАЗАТЕЛЬСТво. Произвольной фиксированной вершине $x^{0} \in V$ поставим в соответствие 1. Так как рассматриваемый граф без ограничения общности можно считать плоским, занумеруем соседние с 1 вершины следуюшим образом: каждой вершине $y \in S(1)$ поставим в соответствие два числа $1, i_{1}$, где $i_{1}=1,2, \ldots,|S(1)|$. Теперь каждой вершине $z \in S\left(1, i_{1}\right)$ сопоставим три числа $1, i_{1}, i_{2}$, где $i_{2}=1,2, \ldots,\left|S\left(1, i_{1}\right)\right|$, и т.д. Таким образом, можно установить взаимно однозначное соответствие между $V$ и $K_{A(\Gamma)}$.

Пусть теперь дано некотороемножество $K_{A}$. Структуру графа на $K_{A}$ определим следующим образом: две последовательности $u, v \in K_{A}$ назовем ближсайшими соседями и соединим ребром, если $u=v, i$ либо $v=u, j$ для некоторого $i$ или $j$. Очевидно, что полученньй таким образом граф будет деревом $\Gamma_{A}$. Предложение доказано.

Таким образом, любое дерево Г мы можем представить как множество вида $K_{A(\Gamma)}$.

Пусть $\xi=\left\{\xi_{1}, \xi_{2}, \ldots, \xi_{n}, \ldots\right\}$ - некоторое разбиение множества $K=K_{A(\Gamma)}$ на классы и $h: K \rightarrow \mathbb{R}$ - произвольное отображение.

ОПРЕДЕЛЕНИЕ 2. Отображение $h$ назовем $\xi$-периодическим, если $h_{x}=h_{i}$ для любого $x \in \xi_{i}, i=1,2, \ldots$.

Пусть $x \in \Gamma$ и ему соответствует $1, i_{1}, i_{2}, \ldots, i_{n} \in K$, тогда, очевидно, $|x|=n$.

Приведем несколько примеров разбиений и периодических отображений.

ПримеР 1. Пусть $\xi^{(m)}=\left\{\xi_{0}, \xi_{1}, \ldots, \xi_{m-1}\right\}$, где $\xi_{i}=\{x \in K:|x|=i(\bmod m), 0 \leqslant$ $i<m\}$. Примером $\xi^{(m)}$-периодического отображения является любая функция вида $h_{x}=h_{i}$, если $x \in \xi_{i}$, где $h_{i} \in \mathbb{R}, i=0,1, \ldots, m-1$.

ПРимеР 2. Пусть $\omega_{x}(j)$ - количество чисел $j$, участвующих в записи $x=1, i_{1}, i_{2}, \ldots$, $i_{|x|}, j \leqslant k_{\Gamma}$. Для любого $B \subseteq N_{\Gamma}=\left\{1,2, \ldots, k_{\Gamma}\right\}$ строим разбиение $\xi_{B}=\left\{\xi_{1}, \xi_{2}\right\}$, где $\xi_{1}=\left\{x \in K: \sum_{j \in B} \omega_{x}(j)\right.$ четно $\}, \xi_{2}=\left\{x \in K: \sum_{j \in B} \omega_{x}(j)\right.$ нечетно $\}$.

ПРимеР 3. Пусть $B_{1}, B_{2}, \ldots, B_{m} \subseteq N_{\Gamma}$. Тогда $\bigcap_{i=1}^{m} \xi_{B_{i}}$ также будет разбиением множества $K$.

4. Описание периодических гиббсовских мер модели Изинга. Пусть $K-$ представление дерева Г. Обозначим

$$
W_{n}=\{x \in K:|x|=n\}, \quad V_{n}=\{x \in K:|x| \leqslant n\}, \quad L_{n}=\left\{l=\langle x, y\rangle \in L: x, y \in V_{n}\right\}
$$


Гамильтониан модели Изинга (неоднородной) на дереве Г имеет вид

$$
H(\sigma)=-\sum_{\langle x, y\rangle \in L} J_{x y} \sigma(x) \sigma(y)
$$

где $J_{x y} \in \mathbb{R}_{+}, \sigma(x) \in\{-1,1\}$ для любого $x, y \in K$.

Пусть $A \subset K$ - конечное подмножество. Обозначим через $\Omega_{A}=\{-1,1\}^{A}$ пространство конфигураций на $A$. Пусть $h_{x}$ - действительнозначная функция $x \in K$. Рассмотрим для каждого $n>0$ меру на $\Omega_{V_{n}}$, определенную формулой

$$
\mu_{n}\left(\sigma_{n}\right)=Z_{n}^{-1} \exp \left\{\beta \sum_{\langle x, y\rangle \in L_{n}} J_{x y} \sigma(x) \sigma(y)+\sum_{x \in W_{n}} h_{x} \sigma(x)\right\}
$$

где $\beta=1 / T$ ( $T>0$ - температура), $\sigma_{n}=\left\{\sigma(x), x \in V_{n}\right\} \in \Omega_{V_{n}}$ и $Z_{n}$ - нормирующий делитель.

Условие согласования для $\mu_{n}\left(\sigma_{n}\right), n \geqslant 1$, определяется равенством

$$
\sum_{\sigma^{(n)}} \mu_{n}\left(\sigma_{n-1}, \sigma^{(n)}\right)=\mu_{n-1}\left(\sigma_{n-1}\right)
$$

где $\sigma^{(n)}=\left\{\sigma(x), x \in W_{n}\right\}$.

ТЕОрема 2. Для того чтобы меры (1) удовлетворяли условию (2), необходимо и достаточно, чтобы для $h_{x}, x \in K$, выполнялось равенство

$$
h_{x}=\sum_{y \in S(x)} f\left(h_{y} ; \theta_{x y}\right)
$$

əде $\theta_{x y}=\operatorname{th}\left(\beta J_{x y}\right) u f(x ; \theta)=\operatorname{arcth}(\theta \operatorname{th} x)$.

ДоКАЗАТЕЛЬСТВО. Необходимость. В силу условия согласования (2) имеем

$$
\frac{Z_{n-1}}{Z_{n}} \sum_{\sigma^{(n)}} \exp \left\{\beta \sum_{x \in W_{n-1}} \sum_{y \in S(x)} J_{x y} \sigma(x) \sigma(y)+\sum_{x \in W_{n}} h_{x} \sigma(x)\right\}=\exp \left\{\sum_{x \in W_{n-1}} h_{x} \sigma(x)\right\},
$$

откуда

$$
\frac{Z_{n-1}}{Z_{n}} \sum_{\sigma(n)} \prod_{x \in W_{n-1}} \exp \left\{\sum_{y \in S(x)}\left(\beta J_{x y} \sigma(x)+h_{y}\right) \sigma(y)\right\}=\prod_{x \in W_{n-1}} \exp \left\{h_{x} \sigma(x)\right\}
$$

Далее,

$$
\frac{Z_{n-1}}{Z_{n}} \sum_{\sigma_{x}^{(n)}:} \prod_{x \in W_{n-1}} \exp \left\{\sum_{y \in W_{n-1}}\left(\beta J_{x y} \sigma(x)+h_{y}\right) \sigma(y)\right\}=\prod_{x \in W_{n-1}} \exp \left\{h_{x} \sigma(x)\right\}
$$


где $\sigma_{x}^{(n)}=\{\sigma(y), y \in S(x)\}, x \in W_{n-1}$. Следовательно,

$$
\frac{Z_{n-1}}{Z_{n}} \prod_{x \in W_{n-1}} \sum_{\sigma_{x}^{(n)}} \exp \left\{\sum_{y \in S(x)}\left(\beta J_{x y} \sigma(x)+h_{y}\right) \sigma(y)\right\}=\prod_{x \in W_{n-1}} \exp \left\{h_{x} \sigma(x)\right\}
$$

Зафиксируем $z \in W_{n-1}$ и перепишем (7) в случаях $\sigma(z)=1$ и $\sigma(z)=-1$. Тогда из полученных равенств следует

$$
\prod_{y \in S(z)} \frac{\exp \left(\beta J_{x y}+h_{y}\right)+\exp \left(-\beta J_{x y}-h_{y}\right)}{\exp \left(-\beta J_{x y}+h_{y}\right)+\exp \left(\beta J_{x y}-h_{y}\right)}=\exp \left(2 h_{z}\right)
$$

откуда

$$
h_{z}=\frac{1}{2} \sum_{y \in S(z)} \ln \frac{\operatorname{ch}\left(\beta J x y+h_{y}\right)}{\operatorname{ch}\left(-\beta J x y+h_{y}\right)}
$$

Из (9) получим (3).

Достаточность. Из (3) получим последовательно $(9),(8),(7),(6),(5),(4)$, что эквивалентно (2). Теорема доказана.

Положим

$$
\Xi=\left\{h=\left(h_{x}, x \in K\right): h_{x}=\sum_{y \in S(x)} f\left(h_{y} ; \theta_{x y}\right)\right\} .
$$

В силу теоремы Колмогорова из теоремы 2 следует, что каждому $h \in \Xi$ соответствует и притом единственная мера Гиббса $\mu$ на $\Omega=\{-1,1\}^{K}$, которая удовлетворяет равенству $\mu\left(\sigma_{n}\right)=\mu_{n}\left(\sigma_{n}\right)$.

Таким образом, задача описания предельных гиббсовских мер сводится к описанию элементов множества $\Xi$. Описание произвольного элемента $\Xi$ - сложная проблема. В данной работе мы ограничимся описанием $\xi$-периодических элементов $\Xi$ для некоторых разбиений $\xi$ множества $K$. Точнее, рассмотрим разбиения $\xi^{(1)}=\{K\}$, т.е. тривиальное разбиение и $\xi_{N_{\Gamma}}$ (см. примеры 1,2$)$.

ОПРЕДЕЛЕНИЕ 3. Меру $\mu$ назовем $\xi$-периодической, если она соответствует $\xi$-периодическому отображению $h \in \Xi ; \xi^{(1)}$-периодическая мера назьвается трансляиионно-инвариантной.

Для описания транслящионно-инвариантной меры из (3) получим

$$
h=\sum_{y \in S(x)} f\left(h ; \theta_{x y}\right), \quad x \in K .
$$

Обозначим $\theta_{x}=\left(\theta_{x y}, y \in S(x)\right)$ и через $g\left(h, \theta_{x}\right)$ обозначим правую часть $(10)$.

Легко проверить следуюшие свойства функции $g$ :

1) $g\left(-h, \theta_{x}\right)=-g\left(h, \theta_{x}\right)$;

2) $\lim _{h \rightarrow+\infty} g\left(h, \theta_{x}\right)=\beta \sum_{y \in S(x)} J_{x y}$;

3) $(d / d h) g\left(0, \theta_{x}\right)=\sum_{y \in S(x)} \theta_{x y}$;

4) $0<(d / d h) g\left(h, \theta_{x}\right) \leqslant \sum_{y \in S(x)} \theta_{x y}$;

5) $\left(d^{2} / d h^{2}\right) g\left(h, \theta_{x}\right)<0, h>0$. 
Рассмотрим уравнение (10) при $x=1 \in K$, т.е.

$$
h=\sum_{i=1}^{\alpha_{1}} f\left(h ; \theta_{1 ; 1, i}\right) .
$$

Из свойств 1)-5) следует, что уравнение (11) имеет единственное решение $h=0$, если $\sum_{i=1}^{\alpha_{1}} \theta_{1 ; 1, i}<1$, и три решения $h=0, \pm h_{*}\left(h_{*}>0\right)$, если

$$
\sum_{i=1}^{\alpha_{1}} \theta_{1 ; 1, i} \geqslant 1
$$

Заметим, что $h=0$ является решением уравнения (10) при любых $x \in K$ независимо от значения $\theta_{x y}$.

Из свойства 1) функции $g$ следует, что если $h_{*}$ является решением $(10)$, то и $-h_{*}$ также является решением (10).

Для того чтобы решения $h_{*}$ уравнения (11) удовлетворяли (10), необходимо и достаточно, чтобы параметры $\theta_{x y}, x \in K \backslash\{1\}, y \in S(x)$, удовлетворяли равенству

$$
h_{*}=\sum_{y \in S(x)} f\left(h_{*} ; \theta_{x y}\right) .
$$

Обозначим через $\mu^{-}, \mu^{0}, \mu^{+}$меры, соответствующие совокупностям величин

$$
\left\{h_{x}=-h_{*}, x \in K\right\}, \quad\left\{h_{x}=0, x \in K\right\}, \quad\left\{h_{x}=h_{*}, x \in K\right\}
$$

соответственно.

Таким образом, доказана следующая

ТЕОРемА 3. Если параметры $\theta_{x y}, x, y \in K$, удовлетворяют условиям (12) $u$ (13), то для неоднородной модели Изинга на дереве $Г$ с представлением $K$ существуют три трансляционно-инвариантные меры: $\mu^{-}, \mu^{0}, \mu^{+}$.

Приступим теперь к описанию $\xi_{N_{\Gamma}}$-периодических гиббсовских мер, т.е. описанию функции $h_{x}, x \in K$, вида

$$
h_{x}= \begin{cases}h_{1}, & \text { если }|x| \text { четно, } \\ h_{2}, & \text { если }|x| \text { нечетно. }\end{cases}
$$

Что касается $h_{1}, h_{2}$, в случае $x=1$ из (3) получим

$$
\left\{\begin{array}{l}
h_{1}=\sum_{i=1}^{\alpha_{1}} f\left(h_{2} ; \theta_{1 ; 1, i}\right), \\
h_{2}=\sum_{j=1}^{\alpha_{1 i}} f\left(h_{1} ; \theta_{1, i ; 1, i, j}\right) .
\end{array}\right.
$$

Подставляя второе уравнение системы (14) в первое, получим

$$
h_{1}=g\left(g\left(h_{1} ; \theta_{1, i}\right) ; \theta_{1}\right) \text {. }
$$


Из свойств функции $g$ следует, что уравнение (15) имеет единственное решение $h_{1}=0$, если

$$
\sum_{i=1}^{\alpha_{1}}\left(\theta_{1 ; 1, i} \sum_{j=1}^{\alpha_{1 i}} \theta_{1, i ; 1, i, j}\right)<1,
$$

и три решения $h_{1}=0, h_{1}= \pm h_{1}^{*}\left(h_{1}^{*}>0\right)$, если

$$
\sum_{i=1}^{\alpha_{1}}\left(\theta_{1 ; 1, i} \sum_{j=1}^{\alpha_{1 i}} \theta_{1, i ; 1, i, j}\right) \geqslant 1 \text {. }
$$

Для любого $i=1,2, \ldots, \alpha_{1}$ предположим вьполнение следующего равенства:

$$
\sum_{j=1}^{\alpha_{1 i}} f\left(h_{1}^{*} ; \theta_{1, i ; 1, i, j}\right)=\sum_{j=1}^{\alpha_{11}} f\left(h_{1}^{*} ; \theta_{1,1 ; 1,1, j}\right) .
$$

Таким образом, если вьполняются (16) и (17), то система уравнений (14) имеет три решения:

$$
h^{0}=(0 ; 0), \quad h^{ \pm}=\left( \pm h_{1}^{*}, \pm h_{2}^{*}\right), \quad \text { где } h_{2}^{*}=\sum_{j=1}^{\alpha_{11}} f\left(h_{1}^{*} ; \theta_{1,1 ; 1,1, j}\right) .
$$

Для того чтобы функция

$$
\pm h_{x}= \begin{cases} \pm h_{1}^{*}, & \text { если }|x| \text { четно, } \\ \pm h_{2}^{*}, & \text { если }|x| \text { нечетно, }\end{cases}
$$

удовлетворяла (3), необходимо и достаточно выполнение следующих условий на параметры $\theta_{x y}$ :

$$
\left\{\begin{array}{l}
h_{1}^{*}=\sum_{y \in S(x)} f\left(h_{2}^{*} ; \theta_{x y}\right), \\
h_{2}^{*}=\sum_{z \in S(y)} f\left(h_{1}^{*} ; \theta_{y z}\right)
\end{array}\right.
$$

для $x \in K \backslash\{1 ; 1, i\}, i=1,2, \ldots, \alpha_{1}$.

Следовательно, при выполнении условий (16)-(18) существуют три $\xi_{N_{\Gamma}}$-периодические функции, удовлетворяюшие (3).

Обозначим через $\mu_{\mathrm{per}}^{-}, \mu^{0}, \mu_{\mathrm{per}}^{+}$соответствуюшие им $\xi_{N_{\Gamma}}$-периодические меры.

Таким образом, доказана следуюшая

Теорема 4. Если параметры $\theta_{x y}, x, y \in K$, удовлетворяют условиям (16)-(18), то для неоднородной модели Изинга на дереве $Г$ с представлением К существуют три $\xi_{N_{\Gamma}}$ периодические меры $\mu^{0}, \mu_{\mathrm{per}}^{ \pm}$.

Заметим, что $\mu^{0}$ совпадает с трансляционно-инвариантной мерой (см. теорему 3 ).

В случае, когда $Г$ совпадает с деревом Кэли, аналогичный результат был получен в работе [7].

Следуюший пример показывает, что условия теоремы 4 проверяемы. 
ПРимеР 4. Пусть $\Gamma=\Gamma_{12}$ есть дерево, удовлетворяющее условию

$$
|S(x)|=\left\{\begin{array}{l}
2, \text { если }|x| \text { четно, } \\
1, \text { если }|x| \text { нечетно. }
\end{array}\right.
$$

Рассмотрим однородную модель Изинга т.е. $J_{x y}=J$. Тогда для описания $\xi_{N_{\Gamma}}$-периодических мер из условия согласования (3) получим

$$
\left\{\begin{array}{l}
h_{1}=2 f\left(h_{2} ; \theta\right) \\
h_{2}=f\left(h_{1} ; \theta\right)
\end{array}\right.
$$

где $\theta=\operatorname{th}(J \beta)$. Решая эту систему относительно $\left(h_{1}, h_{2}\right)$, получим явньй вид решения:

а) при $|\theta|<1 / \sqrt{2}$ система $(*)$ имеет единственное решение $(0,0)$;

б) при $-1<\theta \leqslant-1 / \sqrt{2}$ и $1 / \sqrt{2} \leqslant \theta<1$ система $(*)$ имеет три решения $(0,0)$, $\left( \pm h_{1}^{*}, \pm h_{2}^{*}\right)$, где

$$
h_{1}^{*}=\frac{1}{2} \ln \frac{2 \theta^{2} \sqrt{2 \theta^{2}-1}+\theta^{4}+2 \theta^{2}-1}{\left(1-\theta^{2}\right)^{2}}, \quad h_{2}^{*}=f\left(h_{1}^{*} ; \theta\right) .
$$

5. Случайные блуждания в случайной среде на дереве. В этом пункте, используя для произвольного дерева Г его представление $K$, определим случайное блуждание в случайной среде на Г и рассмотрим задачу невозвратности определенного случайного блуждания.

Для любого $\alpha>0$ будем рассматривать совокупность векторов $\left\{p(x)=\left(p_{y}(x): y \in\right.\right.$ $\left.\left.S_{1}(x)\right), x \in K\right\}$, удовлетворяющую условиям

$$
\sum_{y \in S_{1}(x)} p_{y}(x)=1, \quad p_{y}(x) \geqslant \alpha \quad \text { для всех } x \in K .
$$

Эту совокупность будем назьвать средой и обозначать символом $A$.

ОПРеДЕЛЕНИЕ 4. Случайным блужданием в среде $A$ назовем однородную по времени цепь Маркова $\{x(t), t=0,1, \ldots\}$ с множеством состояний $K$, начальным состоянием $1 \in K$ и матрицей переходных вероятностей $M=\{p(x, y), x, y \in K\}$, заданной следуюшим образом:

$$
p(x, y)= \begin{cases}p_{y}(x), & \text { если } y \in S_{1}(x), \\ 0 & \text { в остальных случаях. }\end{cases}
$$

Случайному блужданию в среде $A$ соответствует вероятностная мера $P_{A}$ на пространстве $X$ траекторий блужданий с $\sigma$-алгеброй $F_{X}$, порожденной цилиндрическими подмножествами $X$, т.е. подмножествами вида

$$
X_{B}=\left\{x=\{x(t), t=0,1,2, \ldots\} \in X: x(t)=x^{*}(t), \text { если } t \in B\right\},
$$

где $x^{*} \in X-$ фиксированная траектория и $B$ - конечное подмножество множества $\{0,1$, $2, \ldots\}$. 
Рассмотрим среду как реализацию некоторого процесса. Тогда $A$ зависит также от параметра $\omega$, изменяющегося на вероятностном пространстве $\left(\Omega, F_{\Omega}, \mu\right)$, где $F_{\Omega}$ - выделенная $\sigma$-алгебра подмножеств $\Omega$ и $\mu$-вероятностная мера.

Среда $A(\omega)=\{p(x)=p(x, \omega), x \in K\}$ назьвается случайной средой.

Обозначим $\Omega_{1}=\Omega \times X, F=F_{\Omega} \otimes F_{X}$, где $F$ - минимальная $\sigma$-алгебра, порожденная множествами вида $A_{1} \times A_{2}, A_{1} \in F_{\Omega}, A_{2} \in F_{X}$.

Меру $\mathscr{P}$ на $\left(\Omega_{1}, F\right)$ определим по формуле

$$
\mathscr{P}\left(B_{1} \times B_{2}\right)=\int_{B_{1}} P_{A}\left(B_{2}\right) d \mu(A),
$$

где $B_{1} \in F_{\Omega}, B_{2} \in F_{X}$.

ОПРЕДЕЛЕНИЕ 5. Случайным блужданием в случайной среде назовем заданный на пространстве $\left(\Omega_{1}, F, \mathscr{P}\right)$ процесс

$$
X(t, \tau)=x(t), \quad \tau=(A, x(\cdot)) \in \Omega_{1}, \quad t \in T=\{0,1, \ldots\} .
$$

Цель этого пункта - изучение предельного поведения траектории $\{X(t, \tau)\}_{t \in T}$. Эта задача в общем случае трудная. Мы рассмотрим некоторый частный случай, когда на среду налагается условие периодичности, при котором задача сводится к изучению случайного блуждания в случайной среде на $\mathbb{Z}=\{\ldots,-2,-1,0,1,2, \ldots\}$.

Будем говорить, что случайное блуждание в случайной среде на $K$ невозвратно, если

$$
\mathscr{P}\left\{\lim _{t \rightarrow+\infty}|X(t, \tau)|=+\infty\right\}=1 .
$$

Пусть $\Phi-$ множество всевозможных отображений $K$ на $\mathbb{Z}$ со следующими свойствами:

(a) $|\varphi(x)| \leqslant|x|$

(b) если $x, y \in K$ соответствуют ближайшим соседям на $\Gamma$, то либо $\varphi(x)=\varphi(y)$ либо $\varphi(x)=\varphi(y) \pm 1$.

Введем для $\varphi \in \Phi, x \in K, z \in \mathbb{Z}$ следующее обозначение:

$$
a_{z}^{\varphi}(x)=\sum_{y \in S_{1}(x): \varphi(y)=z} p_{y}(x) .
$$

ОПРЕДЕЛЕНИЕ 6. Среду $A=\{p(x), x \in K\}$ назовем $\varphi$-периодической, если для всех $x, y \in K$ таких, что $\varphi(x)=\varphi(y)$, вьполняется равенство

$$
a_{z}^{\varphi}(x)=a_{z}^{\varphi}(y) \quad \text { для любого } z \in \mathbb{Z} .
$$

Если среда $A$-периодическая, то в силу (19) заметим, что $a_{z}^{\varphi}(x)$ имеет вид

$$
a_{z}^{\varphi}(x)= \begin{cases}a_{i, i}^{\varphi}, & \text { если } \varphi(x)=i, z=i, \\ a_{i, i \pm 1}^{\varphi}, & \text { если } \varphi(x)=i, z=i \pm 1, \\ 0 & \text { в остальных случаях, }\end{cases}
$$

где $a_{i, i}^{\varphi}, a_{i, i \pm 1}^{\varphi} \geqslant \alpha>0$ и $a_{i, i-1}^{\varphi}+a_{i, i}^{\varphi}+a_{i, i+1}^{\varphi}=1$ для любого $i \in \mathbb{Z}$.

Обозначим $d_{\varphi}=\mathrm{E}_{\mu} \ln \left(a_{0,1}^{\varphi} / a_{0,-1}^{\varphi}\right)$, где $\mathrm{E}_{\mu}$ - математическое ожидание по мере $\mu$. 
Теорема 5. Если существует $\varphi \in \Phi$ такое, что $\Omega$ состоит только из $\varphi$-периодических сред, то

1) $n p u d_{\varphi} \neq 0$

$$
\mathscr{P}\left\{\lim _{t \rightarrow+\infty}|X(t, \tau)|=+\infty\right\}=1
$$

2) $n p u d_{\varphi}=0$

$$
\mathscr{P}\left\{\limsup _{t \rightarrow+\infty}|X(t, \tau)|=+\infty\right\}=1
$$

ДОКАЗАТЕЛЬСТВО. В силу условий (а), (б) на функцию $\varphi$ и в силу $\varphi$-периодичности среды заметим, что функция $\varphi$ индуцирует случайное блуждание $\{\tilde{x}(t), t \in T\}$ с пространством состояний $\mathbb{Z}$, начальным состоянием 0 и матрицей переходных вероятностей $\widetilde{M}=\left\{m_{i j}^{\varphi}\right\}_{i, j \in \mathbb{Z}}$, где

$$
m_{i j}^{\varphi}= \begin{cases}a_{i, j}^{\varphi}, & \text { если } j=i, i \pm 1, \\ 0 & \text { в остальных случаях. }\end{cases}
$$

В силу свойства (а) отображения $\varphi$ имеем

$$
|\tilde{x}(t)| \leqslant|X(t, \tau)| \quad \text { для любого } t \in T \text {. }
$$

Но для $\tilde{x}(t)$ теорема верна (см. [14], [15]). Поэтому из (20) следует, что теорема верна и для $X(t, \tau)$. Теорема доказана.

Заметим, что для применения теоремы 5 необходимо показать существование $\varphi \in \Phi$, для которого вьполняется условие теоремы 5. Эта задача достаточно сложная, и общая конструкция построения такого отображения неизвестна.

Следующий пример показывает, что $\Phi$ непусто.

ПримеР 5. Рассмотрим $\varphi: K \rightarrow \mathbb{Z}$, определенную по формуле

$$
\varphi(x)=\varphi\left(1, i_{1}, i_{2}, \ldots, i_{m}\right)= \begin{cases}-m, & \text { если } i_{1}=1, \\ 0, & \text { если } x=1, \\ m, & \text { если } i_{1} \neq 1 .\end{cases}
$$

Очевидно, что $|\varphi(x)|=|x|$ и $\varphi(x)=\varphi(y) \pm 1$, если $x=y, i$, т.е. $\varphi \in \Phi$.

Заметим, что в [11] на дереве Кэли с использованием его группового представления изучены случайные блуждания в случайной среде, доказан аналог теоремы 5 и построен класс отображений $\varphi$.

В работе [12] для случайного блуждания в случайной среде на дереве Кэли другими методами доказан аналог теоремы 5 без условия периодичности случайной среды.

Автор выражает глубокую признательность проф. Н. Н. Ганиходжаеву за внимание к работе. 


\section{СПИСОК ЦИТИРОВАННОЙ ЛИТЕРАТУРЫ}

[1] Liggett T.M. Multiple transition point for the contact process on a binary tree // Ann. Probability. 1996. V. 24. P. 1675-1710.

[2] Lyons R. The Ising model and percolation on trees and tree-line graphs // Comm. Math. Phys. 1989. V. 125. P. 337-353.

[3] Lyons R. Random walks and percolation on trees // Ann. Probability. 1990. V. 18. P. 931-958.

[4] Pemantle R. The contact process on trees // Ann. Probability. 1992. V. 20. P. 2089-2116.

[5] Ганиходжаев Н. Н. Групповое представление и автоморфизмы дерева Кэли // Докл. АН РУз. 1994. № 4. С. 3-5.

[6] Ганиходжаев Н. Н., Розиков У. А. Описание периодических крайних гиббсовских мер некоторых моделей на дереве Кэли // ТМФ. 1997. Т. 111. №1. С. 109-117.

[7] Розиков У. А. Структуры разбиений дерева Кэли и их применения для описания периодических распределений Гиббса // ТМФ. 1997. Т. 112. № 1. С. 170-175.

[8] Ганиходжаев Н.Н., Розиков У.А. О неупорядоченных фазах некоторых моделей на дереве Кэли // Матем. сб. 1999. Т. 190. № 2. С. 31-42.

[9] Розиков У. А. Описание предельных гиббсовских мер для $\lambda$-моделей на решетках Бете // Сиб. матем. ж. 1998. Т. 39. № 2. С. 427-435.

[10] Розиков У. А. Построение несчетного числа гиббсовских мер неоднородной модели Изинга // ТМФ. 1999. Т. 118. №1. С. 95-104.

[11] Розиков У.А. Критерии возвратности для случайного блуждания в случайной среде на дереве Кэли // Докл. АН РУз. 1998. №9. С. 3-5.

[12] Розиков У. А. Условие невозвратности случайного блуждания в случайной среде на дереве Кэли // Узбекский матем. ж. 1998. № 5. С. 79-85.

[13] Курош А. Г. Теория групп. М.: Наука, 1967.

[14] Летчиков А. В.Критерий применимости центральной предельной теоремы к одномерным случайным блужданиям в случайный средах // Теория вероятностей и примен. 1992. Т. 37. № 3. C. $576-580$.

[15] Solomon F. Random walk in random environments // Ann. Probability. 1975. V. 3. № 1. P. 1-31.

Институт математики АН РУз им. В.И. Романовского

Поступило

E-mail: root@im.tashkent.su

30.11 .2000

Исправленный вариант

05.02 .2002 